\title{
Chronic Myeloid Leukemia Data at ASH 2021: A Podcast on Patient Unmet Needs and Later-Line Treatment Developments
}

Massimo Breccia

Received: December 6, 2021 / Accepted: January 14, 2022 / Published online: January 31, 2022

(c) The Author(s) 2022

Keywords: Chronic myeloid leukemia; CML; Hematology; Later-line treatment; Oncology; Patient unmet needs; Podcast; TKIs; Tyrosine kinase inhibitors

\section{PODCAST TRANSCRIPT}

Lydia Alborn (LA): Managing Editor for Advances in Therapy.

Massimo Breccia (MB): Department of Translational and Precision Medicine, Sapienza University of Rome.

You are listening to an Adis podcast.

LA: Hi everyone and welcome to the Adis rapid plus podcast series. Today we will be focusing on chronic myeloid leukaemia and the key data presented at the ASH 2021 American Society of Hematology Conference. Joining us today is Dr. Massimo Breccia from the Department of Translational and Precision Medicine at the University of Rome. Thank you so much for

Supplementary Information The online version contains supplementary material available at https:// doi.org/10.1007/s12325-022-02048-x.

M. Breccia $(\square)$

Hematology, Department of Translational and

Precision Medicine, Policlinico Umberto I, Sapienza

University, Rome, Italy

e-mail: breccia@bce.uniroma1.it being here and for sharing your insights with us today. Massimo will be taking us through the top highlights of the CML (chronic myeloid leukemia) data from the ASH 2021 Conference, whilst also giving specific focus to patient unmet needs and later-line therapies for the disease.

LA: So, Massimo, there's a lot of important data this year but before we cover that, could you maybe talk us through what the main unmet needs are for patients living with CML?

MB: Thank you for your question. So unmet needs for CML patients who failed at least two lines of treatment are still a matter of discussion. Sequential use of tyrosine kinase inhibitors (TKIs) is associated with a decreased probability of response and worse overall survival. A second line of treatment with a secondgeneration TKI rescued about $45-50 \%$ of patients. We also have to consider that all available TKIs have off-target effects that can lead to long-term safety issues and about $20 \%$ of patients discontinued their treatment due to adverse events.

LA: Right so if sequential use of TKIs is associated with poorer treatment response, as you say, are there any factors contributing to these patients becoming clinically resistant to these TKIs?

MB: Yes, sequential treatment induces the emergence of new mutations (for example, T315I or compound mutations). The frequency 
of T315I mutation was reported ranging between $3 \%$ and $15 \%$. And as you know, currently the unique available option is ponatinib and allogeneic stem cell transplant. This latter procedure has been suggested by international guidelines in patients with poor response to a frontline second-generation TKI followed by ponatinib, or the emergence of mutant clones poorly responsive to available TKIs, or in case of intolerance to multiple TKIs or with inadequate recovery of normal hematopoiesis [1].

LA: It's clear then that novel therapy options for clinically resistant patients are essential. So with this in mind, what new developments have actually been reported for third-line treatment in CML at ASH 2021?

MB: So asciminib, for example, is the first example of [an] allosteric TKI active on [the] myristoilic site of ABL. It has been recently approved by the FDA based on the results of the randomized ASCEMBL trial that tested the drug at the dose of $40 \mathrm{mg}$ BID versus bosutinib at the starting dose of $500 \mathrm{mg}$ in patients in third line.

The results of this trial were updated at 48 weeks of treatment at the last ASH meeting so the cumulative incidence of major molecular response (MMR) was still in favour of asciminib (33.2\%) compared to bosutinib (18.6\%); and the MR4 and MR4.5 rates were $14.0 \%$ and $9.6 \%$ with asciminib and $6.6 \%$ and $2.6 \%$ with bosutinib, respectively. The most common adverse events leading to treatment discontinuation included thrombocytopenia (3.2\%) and neutropenia $(2.6 \%)$ in the asciminib arm and indeed increased alanine aminotransferase (5.3\%) and neutropenia (3.9\%) in the bosutinib arm [2].

Also compassionate use of this drug was activated all over the world and first data presented at the ASH meeting. Thirty-nine patients from the Russian group received asciminib for at least 3 months were reported. The median age was 54 years and 23 were in chronic phase, with $59 \%$ of patients that were mutated and $31 \%$ were T315I mutated. More than $60 \%$ of these patients were heavily pre-treated with more than four lines of therapy and 44\% received previously ponatinib. So $32 \%$ of them achieved a complete cytogenetic response and $34 \%$ an MMR. And patients who started asciminib with a low residual disease burden achieved optimal responses [3].

LA: Were similar results found elsewhere with asciminib?

MB: Yes, also the Spanish experience was reported. Forty-nine patients received the drug for a median time of 11.6 months. The median age was 64 years and 48 were in chronic phase. Again, more than $30 \%$ were mutated and $20 \%$ were T315I mutated. More than $90 \%$ in this experience previously received more than three TKIs and 36\% received ponatinib. More than $40 \%$ of these patients achieved and/or maintained a complete cytogenetic response and $21 \%$ MMR, with fatigue, joint pain and nausea that were the most common reported side effects [4].

LA: Well thank you for that. So, other TKIs are still in fairly early phases; could you maybe talk us through some of the data presented for these?

MB: Yeah, another TKI is vodobatinib, a third-generation TKI with limited off-target effects, actually tested in a phase 1 study. Fiftytwo patients were enrolled, 41 in a dose escalation and 11 in the dose expansion part. Thirtyone patients were resistant and $46 \%$ received more than four TKIs, with 22 patients pre-treated with ponatinib and 20 patients mutated at baseline. So 42 patients were evaluable for response and 24 patients achieved and/or maintained a complete cytogenetic response, while 15 patients achieved an MMR. Most common side effects included thrombocytopenia in 33\% of patients, cough in 19\%, anaemia and diarrhoea in $17 \%$, with only ten patients that presented cardiovascular treatment-emergent adverse events [5] but only one seems to be related to the drug.

The Chinese group updated the results of another TKI, olverembatinib, previously known as HQP1351. A phase 1 study enrolled 101 patients, 86 in chronic phase and 15 in accelerated phase. Again, more than $80 \%$ of these patients received previously two lines of treatment and $62 \%$ harboured the T315I mutation. Overall, $66 \%$ of patients achieved a complete cytogenetic response, 53\% an MMR. In the setting of T315I mutated patients, $78 \%$ achieved a complete cytogenetic response and $71 \%$ a major 
molecular response. The most common side effects reported in the phase 1 study were thrombocytopenia in $77 \%$ of patients and skin hyperpigmentation in $86 \%$ [6].

Another two phase 2 studies were also updated for this drug: both enrolled T315I mutated patients but ponatinib-naïve. Of 41 patients in chronic phase, 32 of them completed 12 cycles and $56 \%$ of them achieved an MMR. Again, the most common side effects were thrombocytopenia in $70 \%$ of cases and skin hyperpigmentation in 56\%. Twenty-three patients were enrolled in accelerated phase, 14 completed 12 cycles and $39 \%$ of patients achieved an MMR. Even in accelerated phase, thrombocytopenia and skin pigmentations were reported in $73 \%$ and $69 \%$ of patients, respectively. In advanced phases of disease were also reported metabolic side effects such as proteinuria, hypocalcaemia and hypertriglyceridemia in $56 \%$ of patients [7].

PF-114 is the other drug, a fourth-generation TKI based on the scaffold of ponatinib. The Russian group updated the results of a phase 1 dose-escalation study. The aim was to determine the maximum tolerated dose (MTD) and the dose-limiting toxicity. Fifty-one patients were enrolled, 46 in chronic phase, 16 patients T315I mutated. Twenty-two percent of patients achieved a complete cytogenetic response and $15.6 \%$ a major molecular response. But the MTD was determined at $600 \mathrm{mg}$ due to the occurrence of grade 3 skin psoriasis-like alterations. No cardiovascular side effects were reported nor ankle-brachial index deviations during the study [8].

LA: That's a brilliant overview of early phase but has anything been presented in perhaps later phases from other trials?

MB: Yeah, the final analysis of the phase 4 BYOND study was detailed. The study showed the management of bosutinib in patients beyond the second line of treatment at the starting dose of $500 \mathrm{mg} /$ day and with the primary endpoint of a confirmed major cytogenetic response at 1 year. Of 163 patients enrolled, $48 \%$ still receive the treatment after a median follow-up of 47.8 months. About $26 \%$ of patients discontinued due to the adverse events and, in fact, the median dose intensity was $300 \mathrm{mg} /$ day with more than $70 \%$ of patients that required a dose reduction. The complete cytogenetic response rate, considering patients who achieved or maintained the response, was $81 \%$. The major molecular response rate was $71.8 \%$ with an overall survival of $88 \%$. Only two patients died [from] the disease [9].

LA: It's really interesting then to see the adverse event data being reported at all of these trials, especially considering their implications on things such as treatment discontinuation. In that respect, has dose optimisation been reported in any other major trials?

MB: Yes, dose optimization was reported for ponatinib in the OPTIC trial. Patients in chronic phase in third or subsequent lines of treatment were initially randomized to three different doses of ponatinib $(45,30$ or $15 \mathrm{mg}$ ) but with a possible de-escalation to $15 \mathrm{mg}$, if started at an increased dose of 45 or $30 \mathrm{mg}$ after the achievement of the primary endpoint (that is less than $1 \% B C R / A B L 1$ ratio at 12 months). The starting dose of $45 \mathrm{mg}$ and the subsequent deescalation to $15 \mathrm{mg}$ was associated to the maximum benefit in terms of efficacy not only in the setting of T315I mutated patients but also with other mutations or in patients who started with a lack of a complete hematologic response. During the last ASH meeting, a subanalysis of the OPTIC trial was presented with response rate according to the baseline $B C R / A B L 1$ level and mutation status. At baseline, $84.1 \%$ of patients had a very high disease burden (more than $10 \% B C R-A B L 1{ }^{I S}$ ratio); nearly $24 \%$ had a T315I mutation and $17.0 \%$ had a mutation different from T315I, with nearly 58\% without mutations. Patients with T315I mutation had the highest primary endpoint (so 60\% by 3 years) with the $45 \mathrm{mg}$ compared with the other cohorts. Patients with more than $10 \%$ of ratio baseline achieved a clinical benefit if started with $45 \mathrm{mg} /$ day [10].

And the efficacy outcomes were generally comparable or better in the OPTIC trial when compared with the previous PACE trial, including the achievement of less than $1 \% B C R$ $A B L 1^{I S}$ response by 24 months. In the PACE trial, 52\%; in the OPTIC trial, 56\%; even according to the 2-year progression-free survival 


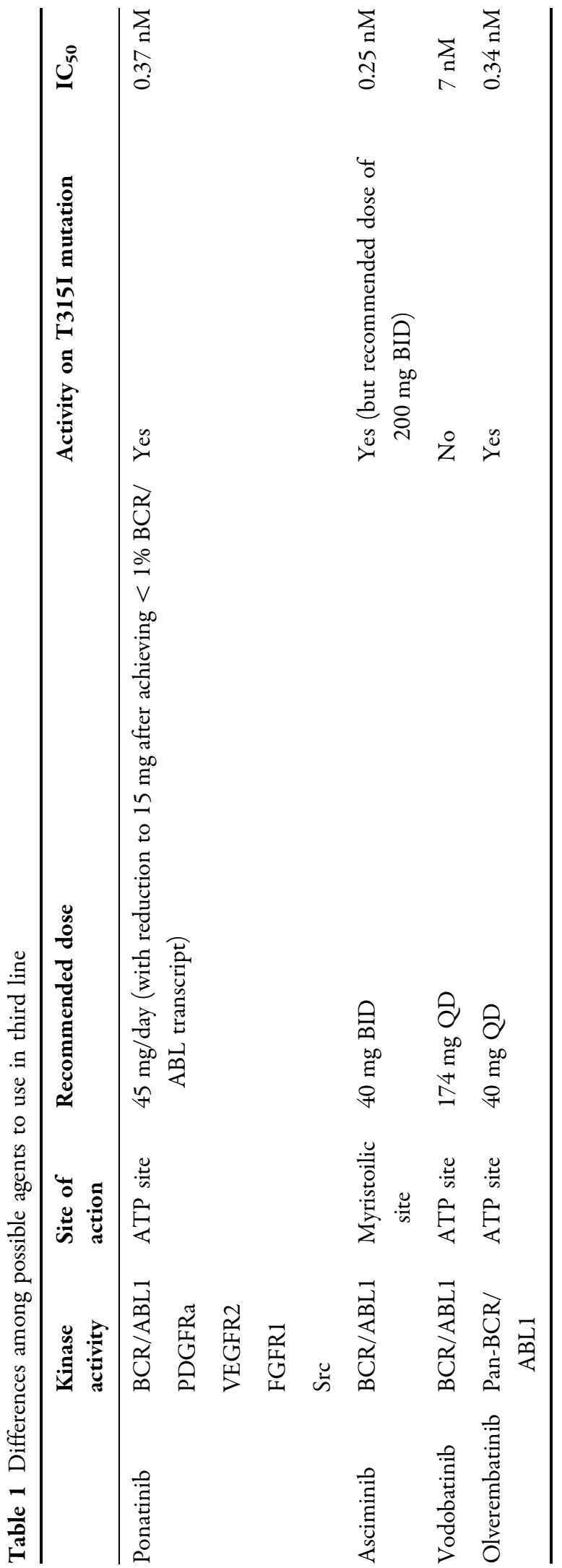

(68\% in PACE vs $80 \%$ in the OPTIC trial) and 2 -year overall survival ( $86 \%$ in the PACE trial; $91 \%$ in the OPTIC trial). So dose reductions due to the adverse events occurred in $82 \%$ of patients in the PACE and $46 \%$ in the OPTIC trial and a $60 \%$ reduction in relative risk for arterial occlusive events in OPTIC trial vs PACE trial was observed [11].

LA: So it's really encouraging then to see all of these developments for potential later-line CML treatments coming out. But what does this mean in terms of patient outcomes moving forwards? Are these adverse events you mentioned, for example, a cause for concern or are the options in general looking quite promising?

MB: Considering the subset of patients resistant or intolerant to several treatments, the results of these new agents seem promising, but I believe it is too early to define an algorithm of treatment, even according to the type of resistance and type of mutations (Table 1). Most of the drugs are very selective and the toxicity seems to be limited to few events with a reduced rate of grade $3 / 4$. And until now off-target effects were not reported.

LA: That's a really fantastic summary of the key data that's come out of ASH 2021. Thank you so much, Massimo, for joining us again and I really hope this will be helpful and interesting for all of our listeners!

MB: Thank you, thank you very much.

You can listen to more podcasts by subscribing to Adis Rapid+ podcast with your preferred podcast provider, or by visiting the website. A full list of declarations, including funding and author disclosure statements, can also be found on the journal website.

\section{DIGITAL FEATURES}

This article is published with digital features, including a podcast audio and slide deck, to facilitate understanding of the article. To view digital features for this article go to https://doi. org/10.6084/m9.figshare.18394235. 


\section{ACKNOWLEDGEMENTS}

Funding. This podcast has been developed through an educational grant from Novartis. The authors were selected by the journal, and the content of the podcast was developed independently by the author and the journal Editor. The Rapid Service and Open Access Fees were also funded by an educational grant from Novartis.

Authorship. Massimo Breccia meets the International Committee of Medical Journal Editors (ICMJE) criteria for authorship for this article, takes responsibility for the integrity of the work as a whole, and has given approval for this version to be published.

Disclosures. Massimo Breccia is on the Editorial Board of Advances in Therapy but has nothing further to disclose.

Compliance with Ethics Guidelines. This article does not contain any studies with human participants or animals performed by the author.

Peer Review. Please note, contrary to the journal's standard single-blind peer review process, as a podcast this article underwent review by a member of the journal's Editorial Board.

Open Access. This article is licensed under a Creative Commons Attribution-NonCommercial 4.0 International License, which permits any non-commercial use, sharing, adaptation, distribution and reproduction in any medium or format, as long as you give appropriate credit to the original author(s) and the source, provide a link to the Creative Commons licence, and indicate if changes were made. The images or other third party material in this article are included in the article's Creative Commons licence, unless indicated otherwise in a credit line to the material. If material is not included in the article's Creative Commons licence and your intended use is not permitted by statutory regulation or exceeds the permitted use, you will need to obtain permission directly from the copyright holder. To view a copy of this licence, visit http://creativecommons.org/licenses/by$\mathrm{nc} / 4.0 /$.

\section{REFERENCES}

1. Hochhaus A, Baccarani M, Silver RT, et al. European LeukemiaNet 2020 recommendations for treating chronic myeloid leukemia. Leukemia. 2020;34(4): 966-84.

2. Mauro MJ, Minami Y, Rea D, et al. Efficacy and safety results for Ascembl, a multicenter, open-label, phase 3 study of asciminib, a first-in-class STAMP inhibitor, vs bosutinib in patients with chronic myeloid leukemia in chronic phase after $>$ 2 prior tyrosine kinase inhibitors: update after 48 weeks. In: ASH 2021: abstract 310.

3. Turkina AG, Shukhov OA, Lomaia E, et al. The first results of asciminib therapy in highly pretreated chronic myeloid leukemia patients under the managed access program (MAP) in Russian Federation. In: ASH 2021: abstract 1483.

4. Perez-Lamas L, Luna A, Boque C, et al. Asciminib in real-life clinical practice, safety and efficacy profile in chronic myeloid leukemia pretreated patients. In: ASH 2021: abstract 2563.

5. Cortes J, Saikia T, Kim D-W, et al. An update of safety and efficacy results from phase 1 dose-escalation and expansion study of vodobatinib, a novel oral BCR-ABL1 tyrosine kinase inhibitor (TKI), in patients with chronic myeloid leukemia (CML) and Philadelphia chromosome positive acute lymphoblastic leukemia (Ph+ ALL) failing prior TKI therapies. In: ASH 2021: abstract 309.

6. Qian J, Shi D, Li, Z, et al. Updated safety and efficacy results of phase 1 study of olverembatinib (HQP1351), a novel third-generation BCR-ABL tyrosine kinase inhibitor (TKI), in patients with TKIresistant chronic myeloid leukemia (CML). In: $\mathrm{ASH}$ 2021: abstract 311 .

7. Qian J, Shi D, Li, Z, et al. Updated results of pivotal phase 2 trials of olverembatinib (HQP1351) in patients with tyrosine kinase inhibitor (TKI)-resistant BCR-ABL T315I-mutated chronic- and accelerated-phase chronic myeloid leukemia. In: ASH 2021: abstract 3598.

8. Turkina AG, Vinogradova O, Lomaia E, et al. PF-114 in patients failing prior tyrosine kinase-inhibitor therapy including BCR-ABL1 T315I. In: ASH 2021: abstract 1482 . 
9. Gambacorti-Passerini C, Brummendorf T, Abruzzese E, et al. Efficacy and safety of bosutinib in previously treated patients with chronic myeloid leukemia: final results from the Byond trial. In: ASH 2021: abstract 1475 .

10. Deininger MW, Apperley JF, Arthur CK, et al. Post hoc analysis of responses to ponatinib in patients with chronic-phase chronic myeloid leukemia (CP-
CML) by baseline BCR-ABL1 level and baseline mutation status in the Optic trial. In: ASH 2021: abstract 307.

11. Jabbour EJ, Deininger MW, Abruzzese E, et al. Dose modification dynamics of ponatinib in patients with chronic-phase chronic myeloid leukemia (CPCML) from the PACE and optic trials. In: ASH 2021: abstract 2550 . 